Found. Phys. Lett. 10, 73-83 (1997)

\title{
NONINVARIANT ONE-WAY SPEED OF LIGHT AND LOCALLY EQUIVALENT REFERENCE FRAMES
}

\author{
F. Selleri \\ Università di Bari - Dipartimento di Fisica \\ INFN - Sezione di Bari \\ I 70126 Bari, Italy
}

We consider an isotropical inertial reference frame ("stationary") and in it a uniformly rotating circular platform of radius $R$. The velocity of light $\tilde{c}$ relative to: the rim of the platform is calculated and found to have values necessarily different from $c$. This $\tilde{c}$ remains the same if $R$ is increased but the peripheral velocity is kept constant. Since by so doing any small piece of the circumference can be considered better and better at rest in a ("moving") inertial system, the velocity of light relative to this system can be deduced. Noninvariant values are obtained and shown to coincide with the predictions of our recently published "inertial transformations".

Key words: special relativity, velocity of light, conventionality.

\section{SPACE AND TIME ON A ROTATING PLATFORM}

The basic idea of the present paper is that every time one learns something new about accelerated reference frames, one learns something new also about inertial frames. In fact the latter frames can always be considered as particular cases of the former ones with zero acceleration. The limit $a \square 0$ does not need to be taken litterally, mathematically. In fact no perfectly inertial frames exist in practice, e.g. because of Earth rotation around its axis, of orbital motion around the Sun, of Galactic rotation. All we know about inertial frames (which is certainly a lot) has actually been learned in frames having a small but nonzero acceleration. Of course the mathematical limit $a \square 0$ in the theoretical schemes can be taken, if one so wishes, but it becomes then essential that it be a smooth limit and that no discontinuities arise between systems with small but nonzero acceleration and inertial systems. From such a point of view our existing theories will be seen to be wanting.

Consider an inertial reference frame $S_{0}$ and assume that it is isotropic. Therefore the one-way velocity of light relative to $S_{0}$ can be taken to have the usual value $c$ in all directions. In relativity the latter assumption is true in all inertial frames, while in other theories only one such frame exists [1]. A laboratory in which 
physical experiments are performed is assumed to be at rest in $S_{0}$, and in it clocks have been synchronised with the Einstein method, that is by using light signals.

In this laboratory there is a perfectly circular platform having radius $R$ which rotates around its axis with angular velocity $\square$ and peripheral velocity $v=\square R$. On its rim we consider a clock $C_{\square}$ and we assume it to be set as follows: When a clock of the laboratory momentarily very near $C_{\square}$ shows time $t_{0}=0$ then also $C_{\square}$ is set at time $t=0$. If the platform were not rotating $C_{\square}$ would always show the same time as the laboratory clocks. When it rotates, however, motion modifies the pace of $C_{\square}$ and the relationship between the times $t$ and $t_{0}$ is then taken to have the general form

$$
t_{0}=t F_{1}(v, a)
$$

where $F_{1}$ is a function of velocity $v$, acceleration $a=v^{2} / R$, and eventually of higher derivatives of position (not shown).

The circumference length is assumed to be $L_{0}$ and $L$, measured from the laboratory $S_{0}$ and on the platform, respectively. Motion can modify length as well, and we assume the relationship

$$
L_{0}=L F_{2}(v, a)
$$

where $F_{2}$ is another function of the said arguments. Notice that the assumed isotropy of the laboratory frame implies that function $F_{1}\left(F_{2}\right)$ does not depend on the position on the rim of the disk where the clock is placed (where the measurement of length is started). It only depends on velocity, acceleration, etc., and these are the same in all points of the border of the rotating circular platform.

We are of course far from ignorant about the nature of the functions $F_{1}$ and $F_{2}$. In the limit of small acceleration and constant velocity they are expected to become the usual time dilation and length conctraction factors, respectively:

$$
F_{1}(v, 0)=\frac{1}{\sqrt{1 \square v^{2} / c^{2}}} \quad ; \quad F_{2}(v, 0)=\sqrt{1 \square v^{2} / c^{2}}
$$

There are even strong indications, at least in the case of $F_{1}$, that the dependence on acceleration is totally absent [2]. All this is however unimportant for our present purposes, because the results obtained below hold for all possible functions $F_{1}$ and $F_{2}$.

\section{VELOCITY OF LIGHT ON ROTATING PLATFORMS}

On the rim of the platform besides the clock $C_{\square}$ there is also a light source $\square$ placed in a fixed position very near $C_{\square}$. Two light flashes leave $\square$ at the time $t_{1}$ of $C_{\square}$ and are forced to move circularly by "sliding" on the internal surface of a cylindrical mirror placed at rest on the platform all around it and very near its border. Mirror apart, the light flashes propagate in the vacuum. The motion of the mirror cannot change the velocity of light, because the mirror is like a source (a "virtual" one) and the motion of a source never changes the velocity of the light signals originating 
from it. Thus, relative to the laboratory, the light flashes propagate with the usual velocity $c$.

The description of light propagation given by the laboratory observers is the following: Two light flashes leave $\square$ at time $t_{01}$. The first one propagates circularly in the sense opposite to the platform rotation and comes back to $\square$ at time $t_{02}$ after a full rotation around the platform. The second one propagates circularly in the same rotational sense of the platform and comes back to $\square$ at time $t_{03}$ after a full rotation around the platform. These laboratory times, all relative to events taking place in a fixed point of the platform very near $C_{\square}$, are related to the corresponding platform times via (1):

$$
t_{0 i}=t_{i} F_{1}(v, a) \quad(i=1,2,3)
$$

Light propagating in the direction opposite to the disk rotation must cover a distance smaller than $L_{0}$ by a quantity $x=\square R\left(t_{02} \square t_{01}\right)$ equalling the shift of $\square$ during the time $t_{02} \square t_{01}$ taken by light to reach $\square$. Therefore

$$
L_{0} \square x=\mathcal{C}\left(t_{02} \square t_{01}\right) \quad ; \quad x=\square R\left(t_{02} \square t_{01}\right)
$$

From these equations one gets:

$$
t_{02} \square t_{01}=\frac{L_{0}}{c(1+\square)}
$$

Light propagating in the rotational direction of the disk must instead cover a distance larger than the disk circumference length $L_{0}$ by a quantity $y=\square R\left(t_{03} \square t_{01}\right)$ equalling the shift of $\square$ during the time $t_{03} \square t_{01}$ taken by light to reach $\square$. Therefore

$$
L_{0}+y=c\left(t_{03} \square t_{01}\right) \quad ; \quad y=\square R\left(t_{03} \square t_{01}\right)
$$

One now gets

$$
t_{03} \square t_{01}=\frac{L_{0}}{c(1 \square \square)}
$$

By taking the difference between (8) and (6) we see that the time delay between the arrivals of the two light flashes back in $\square$ is observed in the laboratory to be

$$
t_{03} \square t_{02}=\frac{2 L_{0} \square}{c\left(1 \square \square^{2}\right)}
$$

This is the well known time delay for the Sagnac effect [3] calculated in the laboratory.

We show next that these relations fix to some extent the velocity of light relative to the disk. In fact (2) and (4) applied to (6) and (8) give

whence

$$
\exists t_{2} \square t_{1} \boxminus F_{1}=\frac{L F_{2}}{c(1+\square)} \quad ; \quad \forall t_{3} \square t_{1} \boxminus F_{1}=\frac{L F_{2}}{c(1 \square \square)}
$$




$$
\frac{1}{\tilde{c}(\square)}=\frac{t_{2} \square t_{1}}{L}=\frac{F_{2}}{F_{1} c(1+\square)} \quad ; \quad \frac{1}{\tilde{c}(0)}=\frac{t_{3} \square t_{1}}{L}=\frac{F_{2}}{F_{1} c(1 \square \square)}
$$

if $\tilde{c}(0)$ and $\tilde{c}(\square)$ are the light velocities, relative to the rim of the disk, for the flash propagating in the direction of the disk rotation, and in the opposite direction, respectively. From (11) it follows that the velocities of the two light flashes relative to the disk must satisfy

$$
\frac{\tilde{c}(\square)}{\tilde{c}(0)}=\frac{1+\square}{1 \square \square}
$$

Notice that the unknown functions $F_{1}$ and $F_{2}$ cancel from the ratio (12). The consequences of (12) will be found in the next section. As of now two important comments must be added.

Firstly, one should realise that (12) does not only give the ratio of the two global light velocities for a full trip around the platform in the two opposite directions, but the local ratio as well: isotropy of space insures that the velocities of light are the same in all points of the rim, and therefore that the average velocities coincide with the local ones.

Secondly, the result (12) holds for platforms having different radius but the same peripheral velocity $v$. Suppose that one builds a set of circular platforms with radiuses $R_{1}, R_{2}, \ldots R_{i}, \ldots\left(R_{1}<R_{2}<\ldots<R_{i}<\ldots\right)$ and makes them spin with angular velocities $\square_{1}, \square_{2}, \ldots \square_{i}, \ldots$ in such a way that

$$
\square_{1} R_{1}=\square_{2} R_{2}=\ldots=\square_{i} R_{i}=\ldots=v
$$

where $v$ is a constant velocity. Obviously (12) applies to all such platforms with the same $\square(\square=v / c)$. The respective centripetal accelerations will be

$$
\frac{v^{2}}{R_{1}}, \frac{v^{2}}{R_{2}}, \ldots \frac{v^{2}}{R_{i}}, \ldots
$$

and will tend to zero with growing $R_{i}$. By the way this is so for all higher derivatives of position: if $\vec{r}_{0}\left(t_{0}\right)$ identifies a point of the rim of the $i$-th platform seen from in the laboratory system, one can easily show that

$$
\left|\frac{d^{n} \vec{r}_{0}}{d t_{0}^{n}}\right|=\frac{v^{n}}{R_{i}^{n ! 1}} \quad(n \geq 1)
$$

Therefore, a little piece of the rim of a platform having peripheral velocity $v$ and very large radius for a short time will be practically equivalent to an inertial reference frame, which is endowed of a velocity only. For all practical purposes the "little piece of the rim of a platform" will be an inertial reference frame. But if this is accepted, it follows also that the velocity of light relative to a moving inertial frame cannot be $c$ (otherwise the left-hand side of (12) should take the value 1).

\section{SPEED OF LIGHT RELATIVE TO INERTIAL FRAMES}


As shown in [1] one can always choose Cartesian coordinate systems in two inertial reference frames $S$ and $S_{0}$ and assume:

(1) that space is homogeneous and isotropic, and that time is also homogeneous;

(2) that relative to $S_{0}$ the velocity of light is the same in all directions, so that Einstein's synchronisation can be used in this frame and the velocity $v$ of $S$ relative to $S_{0}$ can be measured;

(3) that the origins of $S$ and $S_{0}$ coincide at $t=t_{0}=0$;

(4) that planes $\left(x_{0}, y_{0}\right)$ and $(x, y)$ coincide at all times $t_{0}$; that also planes $\left(x_{0}, z_{0}\right)$ and $(x, z)$ coincide at all times $t_{0} ;$ but that planes $\left(y_{0}, z_{0}\right)$ and $(y, z)$ coincide at time $t_{0}=0$ only.

It then follows [1] that the transformation laws from $S_{0}$ to $S$ are necessarily

$$
\begin{aligned}
& \square x=f_{1}\left(x_{0} \square v t_{0}\right) \\
& \theta y=g_{2} y_{0} \\
& \square \\
& \square^{z}=g_{2} z_{0} \\
& t=e_{1} x_{0}+e_{4} t_{0}
\end{aligned}
$$

where the coefficients $f_{1}, g_{2}, e_{4}$, and $e_{1}$ can depend on $v$. If at this point one assumes the validity of the relativity principle (including invariance of light velocity) these transformations reduce necessarily to the Lorentz ones. It was shown in [1] that the most general transformations (16) satisfying the conditions of constant two-way velocity of light and of time dilation according to the usual relativistic factor are such that

$$
f_{1}=\frac{1}{R(\square)} \quad ; \quad g_{2}=1 \quad ; \quad e_{4}=R(\square) \square e_{1} \square c
$$

where $\square=v / c$, and

so that

$$
R(\square)=\sqrt{1 \square \square^{2}}
$$

$$
\begin{aligned}
& \square x=\frac{x_{0} \square \square c t_{0}}{R(\square)} \\
& \square_{\square}=y_{0} \\
& \square z=z_{0} \\
& \square t=R(\square) t_{0}+e_{1}\left(x_{0} \square \square c t_{0}\right)
\end{aligned}
$$

In (18) only $e_{1}$ remains unknown. Length contraction by the factor $R(\square)$ is also a consequence of (18). The inverse speed of light compatible with (18) was shown to be [1]:

$$
\frac{1}{\tilde{c}(\square)}=\frac{1}{c}+\frac{\square \square}{\square c}+e_{1} R(\square) \stackrel{\square}{\square} \cos \square
$$


where $\square$ is the angle between the direction of propagation of light and the absolute velocity $\vec{v}$ of $S$. The transformations (18) represent the complete set of theories "equivalent" to the Special Theory of Relativity (STR): if $e 1$ is varied, different elements of this set are obtained. The Lorentz transformation is found as a particular case with $e_{1}=\square \square / c R(\square)$. Different values of $e_{1}$ are obtained from different clocksynchronisation conventions. In all cases but that of STR such values exclude the validity of the relativity principle, and imply the existence of a privileged frame [1]. For all these theories only subluminal motions are possible $(\square<1)$.

In the previous sections we found a ratio of the one-way velocities of light along the rim of the disk, and relative to the disk itself, different from 1 and given by $\mathrm{Eq}$ (12). Our principle of local equivalence between the rim of the disk and the "tangent" inertial frame requires (12) to apply in the latter frame as well. Eq. (19) applied to the cases $\square=0$ and $\square=\square$ gives

$$
\frac{1}{\tilde{c}(0)}=\frac{1}{c}+\frac{\square \square}{\square c}+e_{1} R(\square) \frac{1}{\square} \quad ; \quad \frac{1}{\tilde{c}(\square)}=\frac{1}{c} \square \frac{\square \square}{\square c}+e_{1} R(\square) \frac{\square}{\square}
$$

This gives

$$
\frac{\tilde{c}(\square)}{\tilde{c}(0)}=\frac{1+\square+c e_{1} R(\square)}{1 \square \square \square c e_{1} R(\square)}
$$

which can agree with (12) for $\square<1$ if and only if

$$
e_{1}=0
$$

The space dependent term in the transformation of time is thus seen to disappear from (18). The same result (21) was obtained in [1] by requiring that the Sagnac effect be explained also on the rotating disk, and not only in the laboratory. See also Ref. [4] for a detailed discussion of the problem both from the special and the general relativistic point of view.

\section{THE INERTIAL TRANSFORMATIONS}

In the previous section we showed that the condition $e_{1}=0$ has necessarily to be used. This generates a transformation different from the Lorentz one [1]:

$$
\begin{aligned}
& \square x=\frac{x_{0} \square \square c t_{0}}{R(\square)} \\
& 日_{\square} y=y_{0} \\
& \square z=z_{0} \\
& \text { 目 } t=R(\square) t_{0}
\end{aligned}
$$

The velocity of light predicted by (22) can easily be found by taking $e 1=0$ in (19): 


$$
\frac{1}{\tilde{c}(\square)}=\frac{1+\square \cos \square}{c}
$$

The transformation (22) can be inverted and gives:

$$
\begin{aligned}
& \square x_{0}=R(\square) \stackrel{\square}{\square}+\frac{\square c}{R^{2}(\square)} t_{\square}^{\square} \\
& \square y_{0}=y \\
& \square \\
& \square z_{0}=z \\
& \square t_{0}=\frac{1}{R(\square)} t \\
& \square
\end{aligned}
$$

Note the formal difference between (22) and (24). The latter implies, for example, that the origin of $S_{0}\left(x_{0}=y_{0}=z_{0}=0\right)$ is described in $S$ by $y=z=0$ and by

$$
x=\square \frac{\square c}{1 \square \square^{2}} t
$$

This origin is thus seen to move with speed $\square c /\left(1 \square \square^{2}\right)$, which can exceed $c$, but cannot be superluminal. In fact a light pulse seen from $S$ to propagate in the same direction as $S_{0}$ has $\square=\square$, and thus [using $\left.(23)\right]$ has speed $\tilde{c}(\square)=c /(1 \square \square)$, which can easily be checked to satisfy

$$
\frac{c}{1 \square \square} \geq \frac{c \square}{1 \square \square^{2}}
$$

One of the typical features of these transformations is of course the presence of relative velocities exceeding $c$. Absolute velocities are instead always smaller than $c$ [1]. It is clear from (25) that the velocity of $S_{0}$ relative to $S$ is not equal and opposite to that of $S$ relative to $S_{0}$. In STR one is used to relative velocities that are always equal and opposite, but this symmetry is a consequence of the particular synchronisation used and cannot be expected to hold more generally [1].

Consider now a third inertial system $S^{\prime}$ moving with velocity $\square^{\prime} c$ and its transformation from $S_{0}$, which of course is given by (18) with $\square \square$ replacing $\square$. By eliminating the $S_{0}$ variables one can obtain the transformation between the two moving systems $S$ and $S^{\prime}$ :

$$
\begin{aligned}
& \square x \square=\frac{R(\square)}{R(\square \square} \theta^{x} \square \frac{\square \square \square}{R^{2}(\square)} c t_{\text {G }}^{\square} \\
& \square y \square=y \\
& \square \\
& \square \square=z \\
& \square t \square=\frac{R(\square \square}{R(\square)} t \\
& \square
\end{aligned}
$$


The name for (22)-(24)-(26) is "inertial transformations". In its most general form (26) the inertial transformation depends on two velocities $\left(v\right.$ and $\left.v^{\prime}\right)$. When one of them is zero, either $S$ or $S^{\prime}$ coincide with the privileged system $S_{0}$ and the transformation (26) becomes either (22) or (24).

A feature characterising the transformations (22)-(24)-(26) is absolute simultaneity: two events taking place in different points of $S$ but at the same $t$ are judged to be simultaneous also in $S^{\prime}$ (and viceversa). Of course the existence of absolute simultaneity does not imply that time is absolute: on the contrary, the $\square$-dependent factor in the transformation of time gives rise to time-dilation phenomena similar to those of STR. Time dilation in another sense is however also absolute: a clock at rest in $S$ is seen from $S_{0}$ to run slower, but a clock at rest in $S_{0}$ is seen from $S$ to run faster so that both observers will agree that motion relative to $S_{0}$ slows down the pace of clocks. The difference with respect to STR exists because a meaningful comparison of rates implies that a clock $T_{0}$ at rest in $S_{0}$ must be compared with clocks at rest in different points of $S$, and the result is therefore dependent on the "convention" adopted for synchronising the latter clocks.

Absolute length contraction can also be deduced from (25): All observers agree that motion relative to $S_{0}$ leads to contraction. The discrepancy with the STR is due to the different conventions concerning clock synchronisation: the length of a moving rod can only be obtained by marking the simultaneous positions of its end points, and therefore depends on the very definition of simultaneity of distant events.

\section{CONCLUSIONS}

Our choice of synchronisation (called "absolute" by Mansouri and Sexl [5]) has been made by considering rotating platforms. The main result of this paper is Eq. (12): the ratio

$$
\square \equiv \frac{\tilde{c}(\square)}{\tilde{c}(0)}
$$

has been calculated along the rim of the platforms and shown, under very general conditions, to have the value (12) which in general is different from unity. Therefore the velocities of light parallel and antiparallel to the disk peripheral velocity are not the same. For SRT this is a very serious problem because a set of platforms with growing radius, but all with the same peripheral velocity, approaches locally better and better an inertial frame. To say that the radius becomes very large with constant velocity is the same as saying that the centripetal acceleration goes to zero with constant velocity. The logical situation is shown in Fig. 1 where one can easily see that SRT predicts a discontinuity at zero acceleration, a sudden jump from the accelerated to the "inertial" reference frames. While all experiments are performed in the real physical world $(a \neq 0, \square=(1+\square) /(1 \square \square))$, our theoretical physics seems so to have gone out of the world $(a=0, \quad \square=1)$ ! 


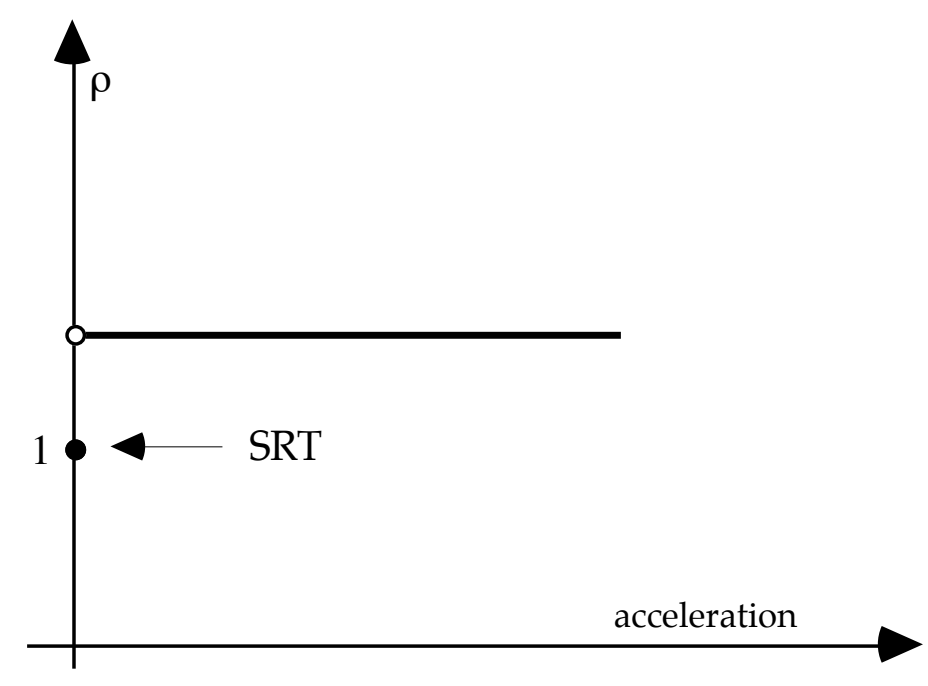

Fig. 1. The ratio $\square=\tilde{c}(\square) / \tilde{c}(0)$ plotted as a function of acceleration for rotating platforms of constant peripheral velocity and increasing radius (decreasing acceleration). The prediction of SRT is discontinuous.

Very probably the above discontinuity is the origin of the synchronisation problems met with the Global Positioning System: after all our Earth is also a rotating platform.

It should be stressed that a noninvariant velocity of light is required for all (but one!) inertial systems. In fact given any such system and a small region of it, it is always possible to conceive a large and rotating circular platform locally at rest in that region, and the result (12) must then apply. Therefore the velocity of light is nonisotropical in all the inertial reference frames with the exception of one $\left(S_{0}\right)$ where isotropy can be postulated.

Finally we must also conclude that the famous synchronisation problem [6] is solved by nature itself: it is not true that the synchronisation procedure can be choosen freely because Einstein's convention leads to an unacceptable discontinuity in the physical theory.

The kinematics of high energy particle interactions was studied in Ref. [7] showing that, in this case, complete equivalence exists between our predictions and those of STR. Therefore the kinematics of high energy processes, the determination of particle masses, and so on, do not require a different analysis from the one successfully carried out up to the present time. In fact energy and momentum are defined in such a way as to coincide numerically with those of STR for all particles and in all inertial frames, once they coincide in the fundamental frame. The coincidence is only numerical (and not also analytical) because the dependence on the "conventional" one-way velocity of the particle is different in the two theories.

\section{REFERENCES}

1. F. Selleri, Found. Phys. 26, 641 (1996).

2. J. Bailey et al., Nature 268, 301 (1977).

3. G. Sagnac, Compt. Rend. 157, 708 (1913); ibidem 1410; 
P. Langevin, Compt. Rend. 173, 831 (1921); 205, 304 (1937);

E. J. Post, Rev. Mod. Phys. 39, 475 (1967).

4. F. Goy and F. Selleri, Time on a rotating platform, Found. Phys. Lett. in print (1997) .

5. R. Mansouri and R. Sexl, Gen.Relat. Gravit. 8, 497 (1977).

6. A.Einstein, RELATIVITY, THE SPECIAL, THE GENERAL THEORY, (Chicago, 1951);

H. Reichenbach, The Philosophy of Space ETime (Dover Publ., New York, 1958).

M. Jammer, "Some fundamental problems in the special theory of relativity", in PROBLEMS IN THE FOUNDATIONS OF PHYSICS, G.

Toraldo di Francia, ed., (Società Italiana di Fisica, Bologna, and North Holland, Amsterdam, 1979).

7. F. Selleri, Found. Phys. Lett. 9, 43 (1996) . 